\title{
Neutron Fluence Estimates Inside the APS Storage Ring During Normal Operation
}

\author{
P.K. Job and J. Alderman \\ Advanced Photon Source \\ Argonne National Laboratory
}

April 2002 


\title{
Neutron Fluence Estimates Inside the APS Storage Ring During Normal Operation
}

\author{
P.K. Job and J. Alderman \\ Advanced Photon Source \\ Argonne National Laboratory
}

April 2002

\section{Introduction}

In an electron storage ring, neutrons are generated as a result of the electron beam interaction with high- $Z$ materials, such as scrapers and collimators [1]. When the energy of the incident electron beam is sufficiently high, it can produce high-energy photons, which subsequently interact with a nucleus, resulting in the emission of nucleons. This interaction is known as a photonuclear interaction. For photons with energies above the typical binding energy of nucleons $(>5-15 \mathrm{MeV})$, photonuclear interaction generally leads to emission of photoneutrons as well as photoprotons. Photonuclear interaction is mainly the result of three specific processes: giant nuclear dipole resonance, quasideuteron production and decay, and intranuclear cascade generated via photopion production.

\section{Photoneutron Production}

Giant nuclear dipole resonance [2] results if the energy of the incident photons is close to the binding energy of the nucleons $(>5-15 \mathrm{MeV})$. In this case, photoabsorption leads to relative displacement of tightly bound neutrons and protons inside the nucleus, resulting in a giant resonance condition. Absorption of the incident photons excites the nucleus to a higher discrete energy state, and the extra energy is emitted in the form of neutrons. For heavy nuclei, the giant resonance decays mainly by neutron emission $(\gamma, n)$. Some contribution from double neutron emission $(\gamma, 2 n)$ is also possible for higher photon energies. Because of the presence of the large Coulomb barrier, proton emission is strongly suppressed for heavy nuclei [2]. For light nuclei, the giant resonance decay is predominantly by single nucleon emission $(\gamma, \mathrm{n})$ or $(\gamma, \mathrm{p})$, with almost equal probability. Giant resonance neutrons are of low energy (1-3 MeV), with a maximum cross section for their production of 1-2 mb/nucleon. The angular distribution of photoneutrons is assumed to be largely isotropic for photon energies below $50 \mathrm{MeV}$ [2].

At incident photon energies $>30 \mathrm{MeV}$, the cross section for giant resonance neutron production decreases rapidly. Above $50 \mathrm{MeV}$, the predominant nuclear photoabsorption mechanism is through quasi-deuteron production and decay [3]. In this process, the incident photon interacts with the dipole moment of a proton-neutron pair inside the nucleus. Neutron-proton pairs inside the nucleus are considered nuclear deuterons. Quasideuteron production gets its name because the proton-neutron pair, or deuteron, does not leave the nucleus. An incident photon couples directly to the electric dipole moment of the quasi-deuteron in the nucleus, causing photodisintegration $(\gamma, n p)$ of the deuteron. A neutron and proton are ejected from the nucleus. The energy range for quasi-deuteron decay neutrons is between 50 and $150 \mathrm{MeV}$, with moderately high-energy neutrons and 
protons emitted from the photodisintegration of the quasi-deuteron. The neutron distribution is found to be only moderately peaked in the forward direction [3].

Photoproduction of pions (photopions) becomes dominant for incident photon energies above $140 \mathrm{MeV}$, where the primary interaction is expected to be between the photon and a single nucleon. The interaction results in the production of one or more $\pi$-mesons or pions. In heavy nuclei, approximately $80 \%$ of the photoproduced pions are reabsorbed by the nucleus leading to the development of an intranuclear cascade [4]. High-energy neutrons, as well as protons and pions, are emitted in the intranuclear cascade process. After the cascade, kinetic energies of the nucleons that did not escape from the nucleus are shared among the nucleons, and the excited nucleus subsequently evaporates emitting low-energy neutrons, protons, and photons [4]. The high-energy particles emitted during an intranuclear cascade process are most likely to be peaked in the forward direction due to the large center of mass forward momentum for the absorption of high-energy photons. However, during the subsequent evaporation of the nucleus, lower energy neutrons are emitted isotropically [4].

Of the three photonuclear interactions discussed, only the giant nuclear dipole resonance process is well understood because the photonuclear interaction cross sections are readily available for this process. Neutron contributions from the other two processes have been estimated as $<10 \%$ for high-energy neutrons above $20 \mathrm{MeV}$ [5]. Therefore, the dominant process of concern for photoneutron production inside the APS storage ring during normal operation is giant nuclear dipole resonance.

\section{Results}

Photoneutron yields corresponding to electron interaction with three metals commonly used in storage ring components were calculated using available cross-section data [6]. Calculations were based on the 7-GeV electron energy of the APS storage ring and only take into account the photoneutron production contribution from the giant nuclear dipole resonance process. Figure 1 provides the calculated photoneutron yield from three target materials as a function of incident electrons. These calculated neutron yields were used to estimate photoneutron production inside the APS storage ring during normal operation.

Two beam loss scenarios inside the APS storage ring — injection beam loss during topup and stored beam loss during normal operation - were considered for the photoneutron production estimates. Yield estimates as a result of stored beam loss were based on an electron beam energy of $7 \mathrm{GeV}$ and a stored beam current of $100 \mathrm{~mA}$. At the stored beam current of $100 \mathrm{~mA}$, the number of stored electrons in the ring is equal to $2.3 \times 10^{12}$. The percentage loss of the total number of electrons in the stored beam, along with neutron yield estimates from infinitely thick targets [6], were used to calculate photoneutron production as a function of stored beam loss. Figure 2 provides the results of the calculation showing photoneutrons emitted as a function of the percentage of stored beam lost on a copper target.

If the typical beam lifetime is eighteen hours, approximately $50 \%$ of the beam will be lost in twelve hours. For stored beam loss on a copper target, this corresponds to 


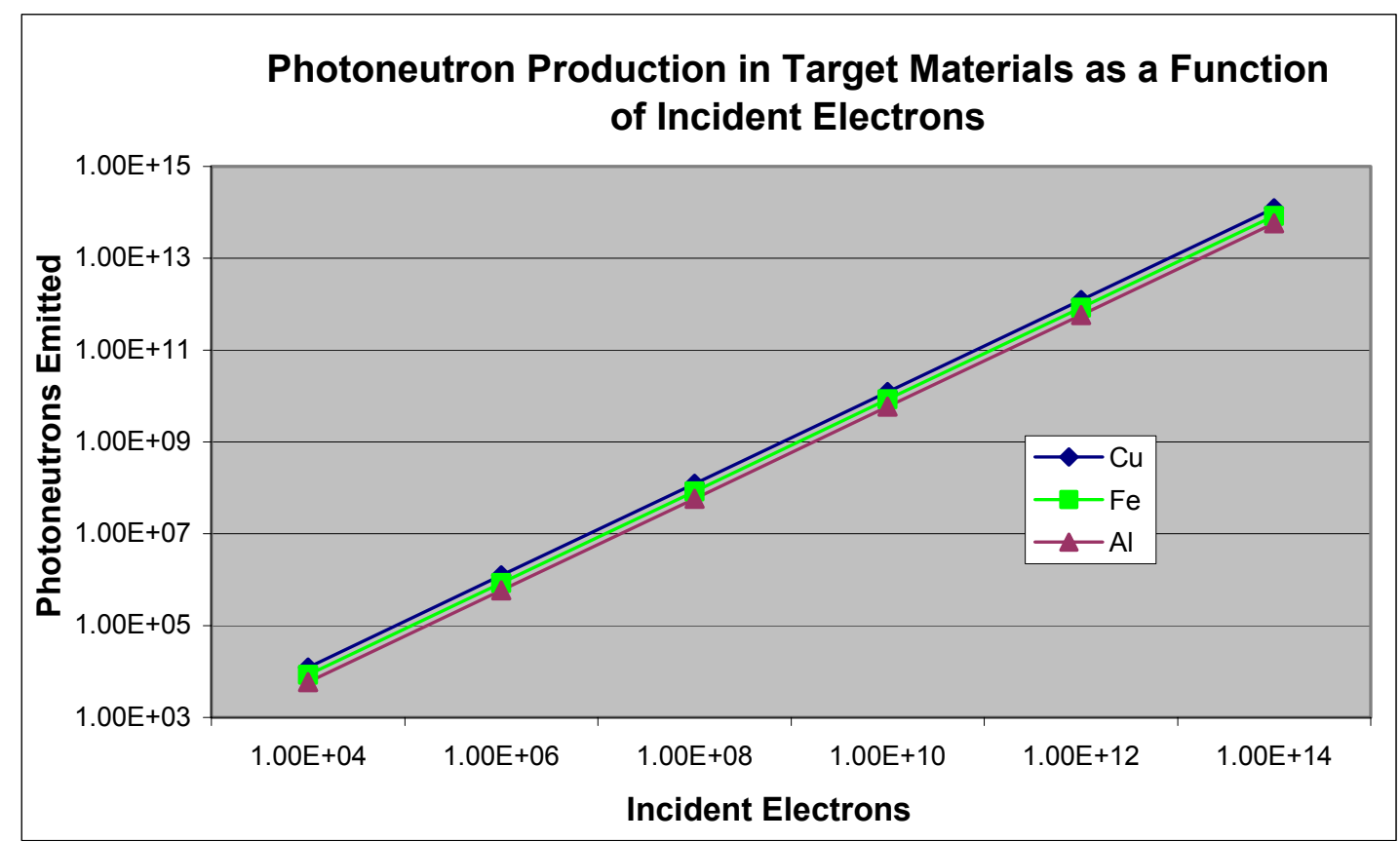

Figure 1: Photoneutron production in target materials as a function of incident electrons (electron energy is $7 \mathrm{GeV}$ ).

approximately $1.4 \times 10^{12}$ neutrons (Figure 2 ). Although at the present time there are 26 insertion device vacuum chambers in the APS storage ring that ultimately serve as constriction points and result in beam loss, a considerable fraction of the stored beam loss occurs at the first smallest constriction point encountered, which is the 5-mm vacuum chamber at sector 3 . Neutron fluence at a given point will be inversely related to the

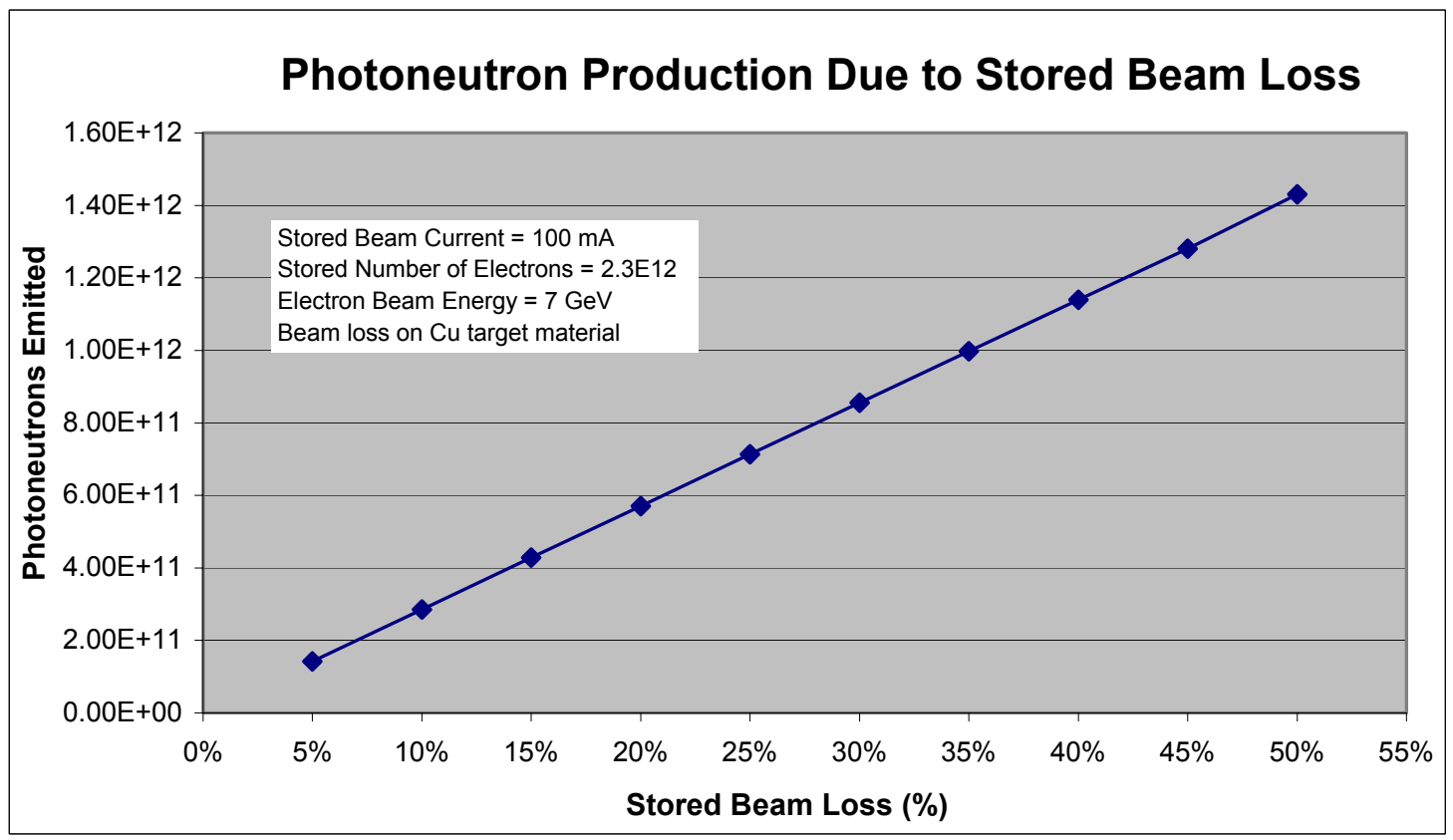

Figure 2: Photoneutron production as a function of the percentage of stored beam loss (copper target). 
square of the distance from a point source. At a distance of $1 \mathrm{~cm}$ from any one of the 26 loss points, assuming all neutrons are generated at a point, this corresponds to $1.1 \times 10^{11} \mathrm{n} / \mathrm{cm}^{2} /$ day. Irradiation in a ${ }^{252} \mathrm{Cf}$ spectrum of sample permanent magnets, similar to those used in APS insertion devices, showed radiation-induced demagnetization for neutron fluence levels of $2 \times 10^{13} \mathrm{n} / \mathrm{cm}^{2}$ and above [7]. For stored beam loss scenarios, using the above fluence rate estimate for a distance of $1 \mathrm{~cm}$, this fluence level could be reached after approximately 180 days. However, under optimal operating conditions, a more even distribution of stored beam loss around the storage ring is expected such that it would take longer to reach these fluence levels at any given loss point.

The second scenario considered for photoneutron production inside the APS storage ring is due to injection beam loss in top-up mode. Photoneutron production estimates were calculated based on a percentage of injection beam loss with a 1.5-nC injection every two minutes. Calculations also took into account the electron beam energy of $7 \mathrm{GeV}$ and the neutron yield estimates from an infinitely thick copper target [6]. Figure 3 provides photoneutron production estimates as a function of the percentage of top-up mode injection beam loss on a copper target. According to the results in Figure 3, for a conservative estimate of $10 \%$ injection loss, photoneutron production in a copper target will be approximately $1.2 \times 10^{9}$ neutrons. At a distance of $1 \mathrm{~cm}$ from the loss point, the corresponding neutron fluence is $9.5 \times 10^{7} \mathrm{n} / \mathrm{cm}^{2} /$ injection. Assuming 720 injections/day based on injections every two minutes during top-up mode, the $2 \times 10^{13} \mathrm{n} / \mathrm{cm}^{2}$ fluence that resulted in radiation-induced demagnetization of permanent magnets would be reached after approximately 300 days of top-up mode injection. In the case of injection beam loss at the APS, a high percentage of beam loss is expected at the first smallest constriction encountered by the injected beam, the $5-\mathrm{mm}$ vacuum chamber at sector 3 . Therefore we would expect these high fluence levels to be reached at this location first.

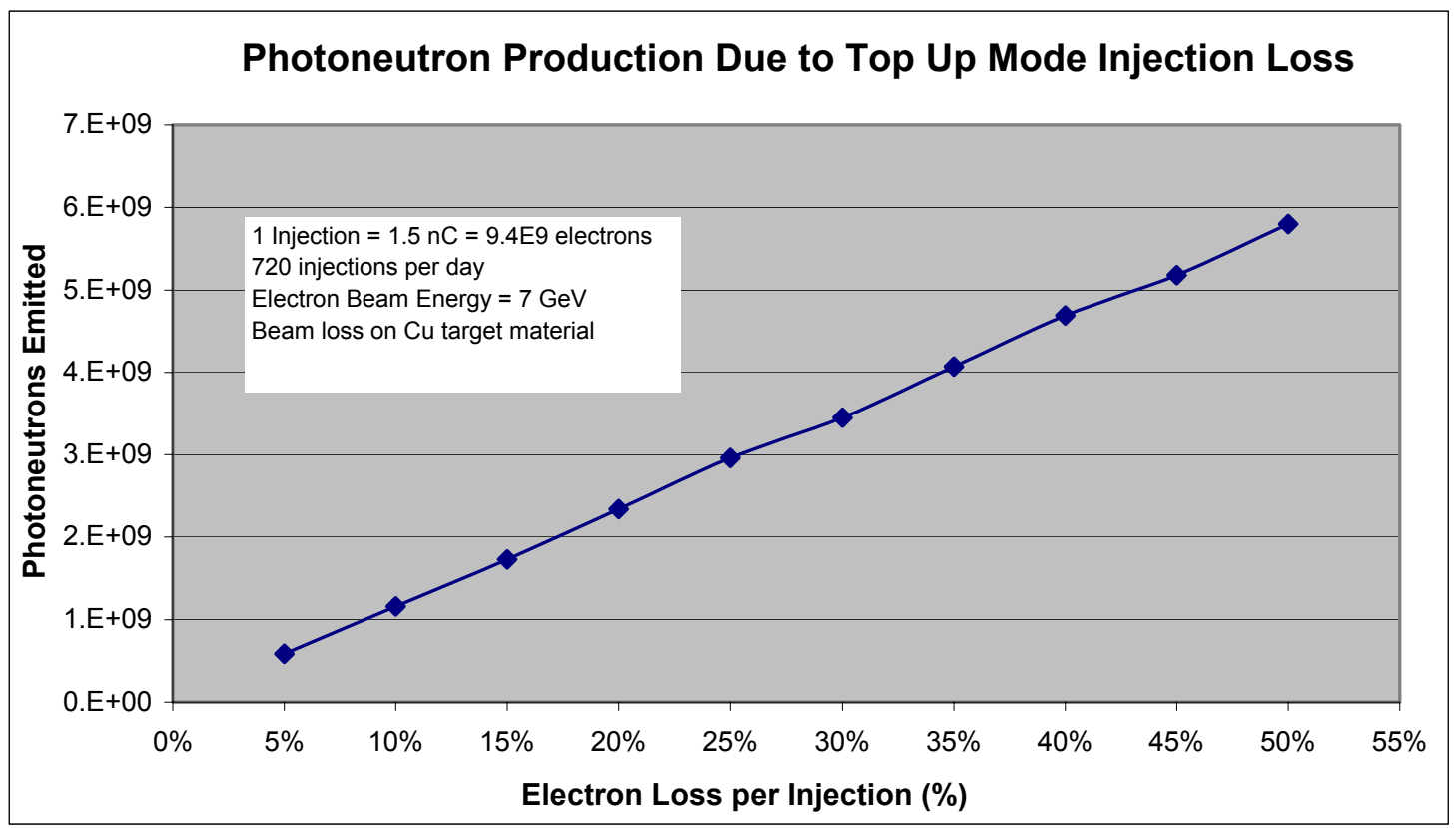

Figure 3: Photoneutron production as a function of the percentage of the top-up mode injection beam loss. 
This report shows that beam losses can be directly related to photoneutron production. For the beam loss scenarios considered, nontrivial neutron fluence levels will be reached during a reasonable period of storage ring operation. The uncertainties associated with these photoneutron estimates are the percentage of beam loss at any given location, the distance of the affected component from the location of the beam loss, and the extent of the electromagnetic shower development in the high- $Z$ material. The examples chosen provide the maximum neutron fluences that may result from the beam loss and the actual fluences may be less than the estimates.

The neutron fluence estimates were only compared to the radiation damage thresholds observed in the permanent magnets [7]. Different storage ring components, like cable insulation, fiber optics, electronics, etc., have potential of damage at varying neutron fluence levels depending on their composition and the radiation resistance [8]. While calculations and estimates are useful, direct measurement of neutron fluence inside the APS storage ring will give a better estimate of photoneutrons produced from beam loss. A program is being initiated to measure photoneutron fluence levels inside the APS storage ring using active and passive devices such as LiF crystals and fission detectors.

\section{References}

[1] M. Pisharody, E. Semones, and P.K. Job, "Dose Measurements of Bremsstrahlung-Produced Neutrons at the Advanced Photon Source," Nucl. Instrum. Methods A 430, 542-558 (1999).

[2] R.D. Evans, The Atomic Nucleus, McGraw-Hill, New York (1955).

[3] J.S. Levinger, “The High Energy Nuclear Photoeffect,” Phys. Rev. 84, 43 (1951).

[4] C.E. Roos and V.Z. Peterson, "Photodissociation of Complex Nuclei at Energies Between the Mesonic Threshold and $1150 \mathrm{MeV}$," Phys. Rev. 124, 1610 (1961).

[5] T.A. Gabriel et al., PICA, An Intranuclear Cascade Calculation for High Energy Photon-Induced Nuclear Reactions, Oak Ridge National Laboratory Report ORNL-4687, Oak Ridge National Laboratory (1971).

[6] W. P. Swanson, Radiological Safety Aspects of the Operation of Electron Linear Accelerators, International Atomic Energy Agency Technical Report, Technical Report Series No. 188, IAEA (1979).

[7] J. Alderman, P.K. Job, R.C. Martin, C.M. Simmons, and G.D. Owen, "Measurement of radiation-induced demagnetization of Nd-Fe-B permanent magnets," Nucl. Instrum. Methods A 481, 9-28 (2002).

[8] P. Beynel, P. Maier, and H. Schonbacher, Compilation of Radiation Damage Test Data, Part III: Materials used around high-energy accelerators, CERN Report CERN 82-10 (November 1982). 